\title{
Some aspects of electron-phonon interaction in the thermal modulation spectra of molecular crystals
}

\author{
B. H. Loo* and A. H. Francis ${ }^{\dagger}$ \\ Department of Chemistry, University of Illinois at Chicago Circle, Chicago, Illinois 60680
}

K. W. Hipps

Department of Chemistry, University of Michigan, Ann Arbor, Michigan 48109

(Received 12 January 1976)

The details of the temperature dependent intensity and linewidth of zero-phonon bands in molecular crystal absorption and emission spectra are considered in relation to the line shape functions observed in thermal modulation spectra. Theory suggests and experiment confirms that in the limit of the Condon and adiabatic approximations for linear electron-phonon coupling, the extraordinary temperature dependence of zero-phonon bands may be utilized to separate them from diffuse background absorption or emission. Qualitative consideration of line broadening from quadratic electron-phonon coupling utilizing a configuration coordinate model suggests that even when these terms dominate the linear interaction, increased resolution may be obtained in a thermal modulation spectrum. The theoretical considerations are illustrated with several experimental examples and some applications of the technique are discussed.

\section{INTRODUCTION}

The identification and characterization of a zero-phonon band or a singularity in the phonon density of states in an optical absorption or emission spectrum is, on occasion, a difficult experimental task. Zero-phonon bands or density of states singularities may be superimposed on a relatively intense and highly structured background absorption or emission and, as a consequence, be partially or completely obscured. More commonly, the zero-phonon line is strongly overlapped by phonon sidebands or inhomogeneously broadened by strain or site distribution. These situations are only partially alleviated by using high quality, strainfree crystals and carrying out experiments at low temperature.

In the following paper (Part II) we are particularly interested in the analysis of the complex phonon sidebands which are a salient feature of the absorption and emission spectra of $p$-benzoquinone pure and chemically mixed crystals. ${ }^{1,2}$ We have found that the details of the structure associated with various features of the optical absorption and emission spectra could, under certain circumstances, be greatly enhanced by thermal modulation spectroscopy ${ }^{3,4}$ and this technique has been utilized extensively in Part II. It is the object of the present paper to make apparent how thermal modulation spectroscopy can be used to develop certain types of spectral features in either absorption or emission from the general background.

In order to discuss the line shapes observed in thermal modulation spectroscopy we obtain first an expression for the temperature dependence of the integrated intensity of the purely zero-phonon band of an electronic transition in the Condon and adiabatic approximations for linear phonon coupling. Additionally, we consider briefly the effects of line broadening due to quadratic phonon coupling using a single configuration coordinate model. We then discuss in terms of the predictions of the simplified model some experimental results abstracted from the thermal modulation absorp- tion and emission spectra of molecular and ionic crystals. Finally, we utilize the model to emphasize some details of practical experimental importance for thermal modulation spectroscopy at cryogenic temperatures.

\section{GENERAL BACKGROUND}

Spectroscopic modulation techniques in general may be conveniently divided into two categories according to whether modulation is achieved internally or externally. In the former case the modulating agent would act directly on some parameter of the sample, while in the latter, more familiar scheme the radiation incident upon the sample would be modulated. In either event, the modulated radiation which is either scattered, transmitted, or reflected by the sample is amplified with phase sensitive detectors or tuned amplifiers. ${ }^{5}$ It is important to emphasize that internal modulation experiments may involve simultaneously a variety of physical processes and the results, therefore, may be more difficult to interpret than those from external modulation experiments. Thermal modulation, for example, produces both a change in the lattice constant and a change in the phonon distribution. The former effect produces a shift in line position, while the latter may result in both a change in linewidth and shift in the line position. ${ }^{6}$

In thermal modulation spectroscopy, one attempts to observe only the changes which occur in an optical emission or absorption spectrum due to small fluctuations in sample temperature. Typically, these changes will involve changes in linewidth, integrated intensity, peak intensity, position, shape, etc. If one is particularly interested in studying the interactions between lattice and electronic excitation, then thermal modulation spectroscopy offers a convenient method of separating many of the quantities of interest in the spectrum from lattice independent effects.

\section{THEORETICAL BACKGROUND}

It is usual to discuss electron-phonon interaction in terms of linear and quadratic phonon coupling terms. 
These arise when the excited state adiabatic potential in which the molecules move is expanded in terms of the ground state lattice normal coordinates. The linear term in the expansion of the adiabatic lattice potential then leads directly to a temperature dependence of the intensity of the zero-phonon line and the multiphonon background or sidebands associated with the zero-phonon band. ${ }^{7}$ The quadratic term produces a temperature dependent width and position of the zero-phonon line.

In order to discuss the line shapes anticipated in a thermal modulation spectrum we must first obtain an expression for the temperature dependence of the line shapes of electronic transitions in solids in several approximations. We wish to evaluate the thermal modulation line shape function for transitions from electronic state $i$ to electronic state $f$ which we write as

$\Delta I(\omega)=[\partial I(\omega) / \partial T] \Delta T$,

$\Delta I(\omega)=B \frac{\partial}{\partial T}\left[A v_{\mathrm{n}} \sum_{\mathrm{m}}\left|\left\langle i \mathrm{n}\left|m_{i f}\right| f \mathrm{~m}\right\rangle\right|^{2} \delta\left(\omega_{f \mathrm{~m}}-\omega_{i \mathrm{n}}-\omega\right)\right] \Delta T$,

where $|i \mathrm{n}\rangle$ and $|f \mathrm{~m}\rangle$ are the vibrational wavefunctions for the initial and final electronic states, respectively, and $A v_{n}$ denotes a thermal average over initial vibrational levels. $B$ is a weakly frequency dependent factor, which in the narrow band approximation ${ }^{8}$ is taken to be a constant.

The problem of the evaluation of the transition moment matrix elements which appear within the thermal average in Eq. (2) has been treated theoretically by numerous authors at varying levels of approximation. The simplest possible model of electron-phonon interaction assumes only a linear electron-phonon coupling and was first considered by Muto, ${ }^{9}$ Huang and Rhys, ${ }^{10}$ and Pekar. ${ }^{11}$ These calculations were later extended to include both linear and quadratic phonon coupling in papers by Lax, ${ }^{12} \mathrm{O}^{\prime}$ Rourke, ${ }^{13}$ and Keil. ${ }^{14}$ Much of the above work is summarized in a review by Dexter ${ }^{8}$ and electron-phonon interaction in the linear coupling approximation has been treated extensively in a book by Rebane. ${ }^{7}$ Considerable work on the effects of the quadratic electron-phonon coupling term have appeared in recent years by Small. ${ }^{6}$ In this section we shall very briefly review some of the aspects of electron-phonon coupling in molecular crystals pertinent to our discussion of line shape changes in thermal modulation spectroscopy. Specifically, we consider linear phonon interaction in the adiabatic and Condon approximations following, principally, the work of Rebane. ${ }^{7}$ In order to qualitatively assess the line shape changes associated with quadratic phonon coupling we will utilize the configuration coordinate model introduced by Seitz, ${ }^{15}$ used extensively by Williams, ${ }^{16}$ and discussed by Lax. ${ }^{12}$

It is usual to describe the molecular impurity in the crystalline lattice in terms of the Hamiltonian operator

$$
H=T_{e}(r)+T_{N}(R)+V(r, R),
$$

where $T_{e}$ is the electronic kinetic energy operator for the impurity electrons, $T_{N}$ is the nuclear kinetic energy operator for both lattice and internal molecular motions, and $V(r, R)$ is the potential energy of the system of lat- tice and impurity nuclei and impurity electrons. It is usual to employ the adiabatic approximation in which the electronic wavefunction depends parametrically upon the nuclear coordinates and is an eigenfunction of

$$
\left(T_{e}+V\right) \phi_{i}(r, R)=E_{i}(R) \phi_{i}(r, R) .
$$

The energy eigenvalues of the electronic wave equation describe the energy surface on which the nuclei move in the adiabatic approximation. In the limit of small vibrational amplitudes, the $E_{i}(R)$ may be expanded in a Taylor series about the equilibrium nuclear positions and, subsequent to a nor mal coordinate transformation will take the form given in Eq. (5),

$$
E_{i}(Q)=E_{i}\left(Q^{0}\right)+\sum_{s} m_{s} \omega_{s}^{2} Q_{s}^{2} / 2
$$

where $m_{s}, \omega_{s}$, and $Q_{s}$ are the effective mass, frequen$c y$, and normal coordinate of the sth normal mode.

The crystal-impurity system vibrational wavefunctions |in) for the $i$ th electronic state may now be obtained as solutions of the vibrational wave equation

$$
\left[T_{N}+E_{i}(Q)\right]|i \mathbf{n}\rangle=\epsilon_{\mathrm{n}}|i \mathrm{n}\rangle
$$

where $\epsilon_{n}$ is the energy of the $n$th vibrational state of the $i$ th electronic state. Solutions of Eq. (6) are of the form

$$
|i \mathbf{n}\rangle=\prod_{s}\left|i n_{s}\right\rangle,
$$

where the functions $\left|i n_{s}\right\rangle$ are harmonic oscillator wavefunctions for the $s$ th independent oscillator normal mode in the $n$th vibrational state and $i$ th electronic state and $\mathbf{n}$ is a vector whose components are the $n_{s}$.

In order to evaluate the matrix elements of the dipole transition moment operator appearing in Eq. (2) it is necessary to obtain the excited state adiabatic vibrational wavefunctions $|f \mathrm{~m}\rangle$. Since the excited state normal coordinates are not in general known, it is usual to expand the excited state adiabatic potential in a Taylor series in the ground state normal coordinates about the ground state equilibrium position. Thus, we obtain for the excited state adiabatic potential

$$
E_{f}(Q)=E_{i f}^{0}+\sum_{s} A_{s} Q_{s}+\sum_{s} \sum_{r} V_{s r} Q_{s} Q_{r} .
$$

If $V_{s r}$ with $s \neq r$ were zero, the vibrational wavefunctions for the excited state would be a product of harmonic oscillator functions differing from the ground state functions in their force constants, due to the quadratic terms with $s=r$, and in their equilibrium positions, due to the terms linear in $Q_{s}$. The cross terms in $Q_{s}$ and $Q_{r}$ arise from a mixing of normal coordinates in the electronic excited state. In certain cases of high site symmetry they may be shown to vanish identically; however, in many cases of interest they will not. These terms will not be considered in the present work.

We shall expand the transition dipole moment in a Taylor series in the ground state normal coordinates and retain only the first two terms, obtaining

$$
M_{i f}(Q)=M_{i f}^{0}+\sum_{s}\left(\theta M / \partial Q_{s}\right)_{0} Q_{s} .
$$

This result may be used to obtain the following expres- 
sion for the line shape function in the adiabatic approximation

$$
\begin{aligned}
I_{i f}(\omega) \equiv & I(\omega) \\
= & B A v_{\mathrm{n}} \sum_{\mathrm{m}} \delta\left(\omega_{f \mathrm{~m}}-\omega_{i \mathrm{n}}-\omega\right)\left[\left|M^{0}\right|^{2}|\langle i \mathbf{n} \mid f \mathrm{~m}\rangle|^{2}\right. \\
& \left.+\sum_{s}\left(\partial M / \partial Q_{s}\right)_{0}\left|\left\langle f \mathrm{~m}\left|Q_{s}\right| i \mathrm{n}\right\rangle\right|^{2}\right]
\end{aligned}
$$

If we restrict our attention to the term remaining in the Condon approximation, we obtain for the intensity and position of the band corresponding to the transition from the state $|i n\rangle$ to the state $|f \mathrm{~m}\rangle$ the following relation $^{7}$ :

$$
\begin{aligned}
I_{\mathrm{am}}(\omega)= & B\left|M^{0}\right|^{2} Z^{-1} \\
& \times \prod_{s} e^{-\hbar \omega_{s} n_{s} / k T}\left|\left\langle i n_{s} \mid f m_{s}\right\rangle\right|^{2} \delta\left(\omega_{f \mathrm{~m}}-\omega_{i \mathrm{n}}-\omega\right),
\end{aligned}
$$

where $Z$ is the partition function for the vibrational states of the initial electronic state. Thus, the line corresponding to an electronic transition from the initial vibronic state characterized by a set of vibrational quantum numbers $\mathbf{n}$ for the independent oscillators to a final vibronic state characterized by a set of vibrational quantum numbers $m$ consists of a single delta function line at frequency $\omega=\omega_{f m}-\omega_{i n}$. The intensity of the zero-phonon line is obtained from Eq. (11) by setting $\mathbf{n}=\mathbf{m}$ and summing over all possible $\mathbf{n}$. That is,

$$
\begin{aligned}
I_{0}(\omega)= & B\left|M^{0}\right|^{2} Z^{-1} \\
& \times \sum_{a} \prod_{s} e^{-\hbar \omega_{s} n_{s} / k T}\left|\left\langle i n_{s} \mid f n_{s}\right\rangle\right|^{2} \delta\left(\omega-\omega_{0}\right) .
\end{aligned}
$$

In the limit of linear electron-phonon coupling and the Condon approximation (12) may be show $^{7}$ to reduce to the following expression:

$$
\begin{aligned}
I_{0}(\omega)= & B\left|M^{0}\right|^{2} \delta\left(\omega-\omega_{0}\right) \\
& \times \exp \left[-\sum_{s}\left(p_{s} / \hbar \omega_{s}\right) \operatorname{coth}\left(\hbar \omega_{s} / 2 k T\right)\right] .
\end{aligned}
$$

We may obtain from Eq. (13) an expression for the thermal modulation line shape function by differentiation with respect to temperature. The finite change in the intensity of the zero-phonon band in the adiabatic and Condon approximations arising from linear electron-phonon coupling is simply

$$
\Delta I_{0}(\omega)=\left\{-\left[I_{0}(\omega) / 2 k T^{2}\right] \sum_{s} p_{s} \operatorname{csch}^{2}\left(\hbar \omega_{s} / 2 k T\right)\right\} \Delta T,
$$

which, if $h \omega_{s} / k T \ll 1$, reduces to

$$
\begin{aligned}
\Delta I_{0}(\omega)= & -B \delta\left(\omega_{0}-\omega\right) \Delta T\left|M^{0}\right|^{2} \\
& \times\left[\sum_{s}\left(2 k p_{s} / \hbar^{2} \omega_{s}^{2}\right)\right] \exp \left[-\sum_{s}\left(2 k p_{s} T / \hbar^{2} \omega_{s}^{2}\right)\right],
\end{aligned}
$$

where $p_{s}=\frac{1}{2} \omega_{s}^{2} m_{s}\left(A_{s} / V_{s s}\right)^{2}$ is the Stokes loss for the $s$ th oscillator.

The ratio of the integrated intensity of the zero-phonon band to the total integrated intensity of the entire system (i.e., zero-phonon band and phonon sidebands) is given by the Debye-Waller factor and depends only upon temperature and the total Stokes loss at the present level of approximation. In the adiabatic and Condon approximations the total integrated intensity of the band is constant and the intensity which is lost from beneath the zero-phonon line with increasing temperature is distributed to the phonon sidebands. Since the phonon sidebands cover a broad spectral region relative to the zero-phonon band, the increase in intensity at a point on the sideband is relatively small compared to the loss in intensity of the zero-phonon band. If the phonon sideband structure contains localized modes or pronounced singularities in the density of states of the band modes, the variation in intensity across the sideband structure will not be uniform. These special points in the frequency spectrum will vary more rapidly with temperature than the general multiphonon background.

If we write the average change in modulated intensity across the bandwidth of the phonon sidebands $\Delta \Omega$ as

$$
\overline{\Delta I}=(1 / \Delta \Omega) \sum_{\mathrm{n} \neq \mathrm{m}}\left[\Delta I_{\mathrm{nm}}(\omega)\right],
$$

where $\left[\Delta I_{\mathrm{am}}(\omega)\right]$ indicates the integrated modulated intensity of a single transition $|i \mathfrak{n}\rangle \rightarrow|f \mathrm{~m}\rangle$. We may utilize the fact that the integrated intensity of the entire band system does not change to obtain the following approximate expression for the change in intensity at a point on the phonon sideband structure:

$$
\overline{\Delta I} \simeq-(\Delta \omega / \Delta \Omega)\left[\Delta I\left(\omega_{0}\right)\right],
$$

where we have used $\Delta \omega$ to designate the monochromator bandpass. The ratio $\Delta \omega / \Delta \Omega$ may be made arbitrarily small experimentally, reducing the background intensity at arbitrary points in the phonon sideband structure to near zero in the thermal modulation spectrum. Points corresponding to zero-phonon bands or singularities in the band phonon density of states, however, will retain considerable intensity in the thermal modulation spectrum.

The greater the width of the phonon sideband structure, the more its effect is suppressed in the thermal modulation spectrum. Therefore, the greater the Stokes losses or the smaller the Debye-Waller factor, the more effective will be the suppression of the multiphonon background in the thermal modulation spectrum. Of course, it is precisely under conditions of weak zero-phonon bands and intense background that one would be most interested in thermal modulation techniques.

Finally, we note that since the total integrated intensity of the band system in the Condon approximation is temperature independent, ${ }^{7}$ the zeroth moment or integrated intensity of the thermal modulation band system must be identically zero. The integration may be performed with particular ease experimentally and provides a sensitive test for the validity of the Condon approximation for individual band systems in the thermal modulation spectrum. This point is discussed more fully in Part II.

\section{QUADRATIC MODES}

There is a close analogy between the shape of the quadratically broadened zero-phonon band in the spec- 
tra of solids and the envelope of the sequence bands observed in the vapor phase spectroscopy of polyatomic molecules. ${ }^{17}$ Sequence structure arises, typically from a series of vibronic transitions associated with one particular vibrational mode $\left(\omega_{i}\right)$, in which the number of vibrational quanta $\left(n_{i}\right)$ remains unchanged, that is, $\Delta n_{i}=\left(n_{i}^{\prime}-n_{i}^{\prime \prime}\right)$ is a constant. The $n_{i}^{\prime}=0=n_{i}^{\prime \prime}=0$ transition in each sequence is referred to as the "parent band" and the spacing between successive sequence bands is determined by the difference between the vibrational frequencies of the sequence forming mode in the upper and lower states. Intensities along sequences are usually found to decrease approximately exponentially with increasing $n_{i}^{\prime \prime}$, roughly in proportion to the Boltzmann factors of the initial vibrational levels. The intrinsic intensities of the sequence members depend upon the vibrational overlap integrals in the relevant normal coordinate.

It is the change in frequency of the lattice modes upon electronic excitation of the impurity center which produce a temperature dependent line broadening in solids. If the temperature dependence of the linewidth is sufficiently great, it might appear that the effect of thermal modulation could only be to obscure any existent sharp structure in the absorption or emission spectrum. One might anticipate that such would be the case when the quadratic terms dominated the linear coupling terms. We shall demonstrate, however, that the thermal modulation line shapes of quadratically broadened zerophonon bands are, in general, narrower than the normal absorption or emission linewidth.

In order to consider the effect of quadratic modes in the thermal modulation spectrum we employ a configuration coordinate model in which we assume that only a limited number of normal modes interact with the electronic excitation and that the cumulative effect of this interaction may be represented by as few as a single schematic coordinate. $\mathrm{Lax}^{12}$ has shown that it is generally possible to treat the problem of electron-phonon interaction in this manner, however, the configuration coordinate will in general not correspond to a real normal coordinate of the lattice-impurity system and the theoretical formulation may require a temperature dependent effective mass for the one dimensional oscillator in order to obtain agreement with experiment.

In the simplified configuration coordinate model, the zero-phonon band and the multiphonon background is composed of a series of closely spaced lines of zero width. The zero-phonon band shape function may be written as

$$
\begin{aligned}
I_{0}(\omega)= & B\left|M^{0}\right|^{2} Z^{-1} \\
& \times \sum_{n}|\langle i n \mid f m\rangle|^{2} \exp \left[-\left(n \hbar \omega_{i} / k T\right)\right] \delta\left(n \Delta \omega+\omega_{0}-\omega\right),
\end{aligned}
$$

where $\omega_{0}$ is the zero-point frequency separation between the initial and final electronic states and corresponds to the frequency of the parent line, and $\Delta \omega=\left(\omega_{f}-\omega_{i}\right)$ is the difference between the fundamental frequencies of the single mode in the initial $\left(\omega_{i}\right)$ and final $\left(\omega_{f}\right)$ electronic states. The functions $|i n\rangle$ and $|f m\rangle$ are the lat-

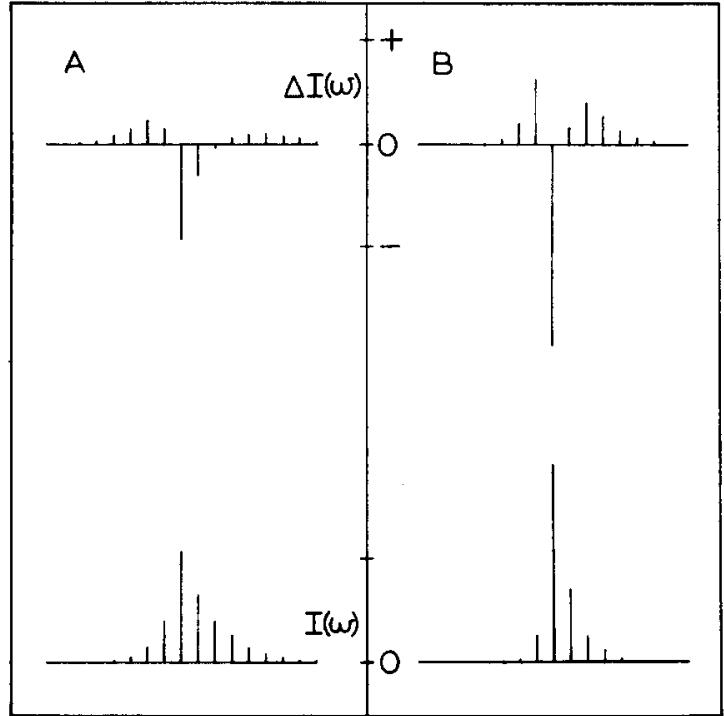

FIG. 1. The spectral line shapes (lower figures) and thermal modulation line shapes (upper figures) obtained for a two coordinate model with (a) $\hbar \omega_{1}=2 \hbar \omega_{2}=k T$ and (b) $1 / 2 \hbar \omega_{1}=\hbar \omega_{2}=k T$.

tice vibrational wavefunctions for the initial and final electronic levels, respectively. The remaining terms have the same meaning as defined previously.

For moderate differences in the ground and excited state force constants the zero-phonon band FranckCondon factors are a slowly varying function of the vibrational index whose general form has been given by Keil $^{14}$ :

$$
\langle i n \mid f m\rangle=1-\left[\left(n^{2}+n+1\right) / 4\right]\left(\Delta \omega / \omega_{i}+\omega_{f}\right)^{2} .
$$

Provided the frequency of the schematic mode does not change too greatly upon electronic excitation we may set $\langle i n \mid f m\rangle=1$ and obtain for the line shape function

$$
\begin{aligned}
I_{0}(\omega)= & B\left|M^{0}\right|^{2}\left(1-e^{-\hbar \omega_{i} / k T}\right) \\
& \times \sum_{n} \exp \left[-\left(n \hbar \omega_{i} / k T\right)\right] \delta\left(n \Delta \omega+\omega_{0}-\omega\right) .
\end{aligned}
$$

Equation (20) is a series of equidistant delta functions displaced to higher or lower frequencies of the parent line as the difference in the normal mode phonon frequency upon electronic excitation is negative or positive, respectively.

The line shape of the zero-phonon band in the thermal modulation spectrum may be obtained from Eq. (20) by differentiation with respect to temperature. For small modulation amplitudes $\Delta T$, the line shape function is given by

$$
\begin{aligned}
\Delta I_{0}(\omega)= & B\left|M^{0}\right|^{2}\left(\hbar \omega_{i} \Delta T / k T^{2}\right) \\
& \times \sum_{n}\left[e^{-\hbar n \omega_{i} / k T} n-(n+1) e^{-(n+1) n \omega_{i} / k T}\right] \\
& \times \delta\left(n \Delta \omega+\omega_{0}-\omega\right) .
\end{aligned}
$$

In Fig. 1 we have illustrated the form of both $I_{0}(\omega)$ and $\Delta I_{0}(\omega)$. We have utilized, in computing the line shapes shown in Fig. 1, a two coordinate model in order to account qualitatively both for modes which increase in frequency upon electronic excitation as well as those which 
decrease. In general, the line shape function for $n$ coordinates is obtained simply as a convolution of the line shape functions for each individual coordinate.

We may make the following general observations about the line shape function given in Eq. (21). At the present level of approximation, having neglected the dependence of the Franck-Condon factors on the vibrational index, the integrated intensity of the zero-phonon band is temperature independent and the integrated intensity of the thermal modulation zero-phonon band is, therefore, zero.

There are temperature invariant points in the thermal modulation spectrum to higher or lower frequency of the parent line as $\Delta \omega$ is positive or negative, respectively.

Within the zero-phonon band, the parent line, corresponding to the single delta function transition for which $n=m=0$ will vary the most rapidly with temperature; a fact of little consequence in conventional spectra since the components of the quasiline are not individually resolved. In the thermal modulation spectrum, however, the rapid variation with temperature within the quadratically broadened zero-phonon band in the region of the parent line accentuates this region of the line. Since each quadratic mode contributes one delta function component to the parent line the effect is multiplied by additional quadratic modes. If the rise in the average temperature of the lattice during the thermal modulation spectrum is not too great, the thermal modulation line shape function is a more rapidly varying function of frequency than the normal line shape function and may appear narrowed about the parent line.

\section{FORBIDDEN ELECTRONIC TRANSITIONS}

When the electronic transition is forbidden by dipole selection rules, the first term of Eq. (10) vanishes and the transition dipole strength is determined by the dependence of the transition dipole moment operator upon the lattice coordinates given by the remaining term. The second term of Eq. (10) represents, therefore, deviations from the Condon approximation. In the linear phonon coupling approximation, Rebane ${ }^{7}$ has shown that the integrated intensity of the entire forbidden band will vary as

$$
[I(\omega)]=\sum_{s}\left(B \hbar / 2 m \omega_{s}\right)\left(\partial M / \partial Q_{s}\right)_{0}^{2} \operatorname{coth}\left(h \omega_{s} / 2 k T\right) .
$$

As in the case of the dipole allowed transition, the integrated intensity of the dipole forbidden zero-phonon band is expected to be an exponentially decreasing function of temperature.

\section{EXPERIMENTAL CONSIDERATIONS}

We shall define the thermal modulation efficiency ${ }^{5}$ as the ratio of the thermal modulation amplitude $\Delta T$ to the average temperature of the sample $T$. The thermal modulation signal $\Delta I(\omega)$ is an increasing function of the thermal modulation efficiency defined in this manner and one should attempt to maximize this parameter experimentally. This does not ultimately mean, however, using the largest possible heat pulse amplitude $\Delta T_{h}$, since for a fixed modulation frequency increasing the heat pulse amplitude produces a corresponding rise in the average temperature which quickly reduces $\Delta I(\omega)$. The sample dimensions and the heater configuration have a pronounced effect on the magnitude of $\Delta T / \bar{T}$, which can be obtained experimentally.

Typically, thermal modulation of the lattice temperature is achieved by passing a heating current through a thin resistive film to which the sample is strongly bonded. The samples are prepared in the form of thin slabs with thickness several orders of magnitude less than cross-section dimensions, circumstances under which heat transfer is essentially one dimensional and perpendicular to the surface. We assume that the temperature of the sample is uniform and that the rate of heat transfer from the sample to the bath is proportional to the rise above ambient temperature. This is equivalent to assuming that cooling of the sample is governed by the Kapitza resistance ${ }^{18}$ at the sample-bath boundary, rather than by the rate of heat diffusion within the sample itself. This approximation is valid for single crystal samples of moderate thermal conductivity at cryogenic temperatures, provided the boundary temperature difference is small. With the above assumptions, we may derive the following expression ${ }^{3}$ for the thermal modulation amplitude $\Delta T$ :

$$
\Delta T=\Delta T_{h} \tanh (1 / 2 \tau f),
$$

where $f$ is the modulation frequency, $\Delta T_{h}$ is the amplitude of the temperature change at the heater, and $\tau$ is the boundary limited thermal relaxation time given by

$$
\tau=R_{K} S L \rho,
$$

where $R_{K}$ is the Kapitza resistance, $S$ is the specific heat, $\rho$ the density of the sample, and $L$ is the sample thickness.

Uniformity of the internal temperature is important in order to avoid inhomogeneous broadening of the line shapes. The temperature of the sample will be approximately uniform provided that the boundary limited relaxation time is long compared to the time required to obtain internal equilibrium given by:

$$
\tau^{\prime}=S L^{2} \rho / k
$$

where $k$ is the thermal conductivity of the material. For reasonable values of the parameters involved at liquid helium temperatures $\tau^{\prime} \sim 10^{-6} \mathrm{sec}$ in a $1 \mathrm{~mm}$ thick sample. There are few values of $R_{K}$ for dielectric solids; however, from the data available a reasonable estimate $^{18}$ for $R_{K}$ at $2^{\circ} \mathrm{K}$ is on the order of $10^{\circ} \mathrm{K}-\mathrm{cm}^{2} / \mathrm{W}$ and the boundary limited thermal relaxation time for a $1 \mathrm{~mm}$ thick sample becomes $\tau \sim 10^{-4} \mathrm{sec}$.

\section{EXPERIMENT}

We have utilized an absorption band from the spectrum of single crystal $p$-benzoquinone to illustrate the development of spectral detail in the thermal modulation spectrum. The band group shown in the lower most trace of Fig. 2 has been identified tentatively ${ }^{19}$ as a vibronic origin of the ${ }^{3} A_{u}-{ }^{1} A_{g}$ transition, although the assignment of the transition is irrelevant for present 


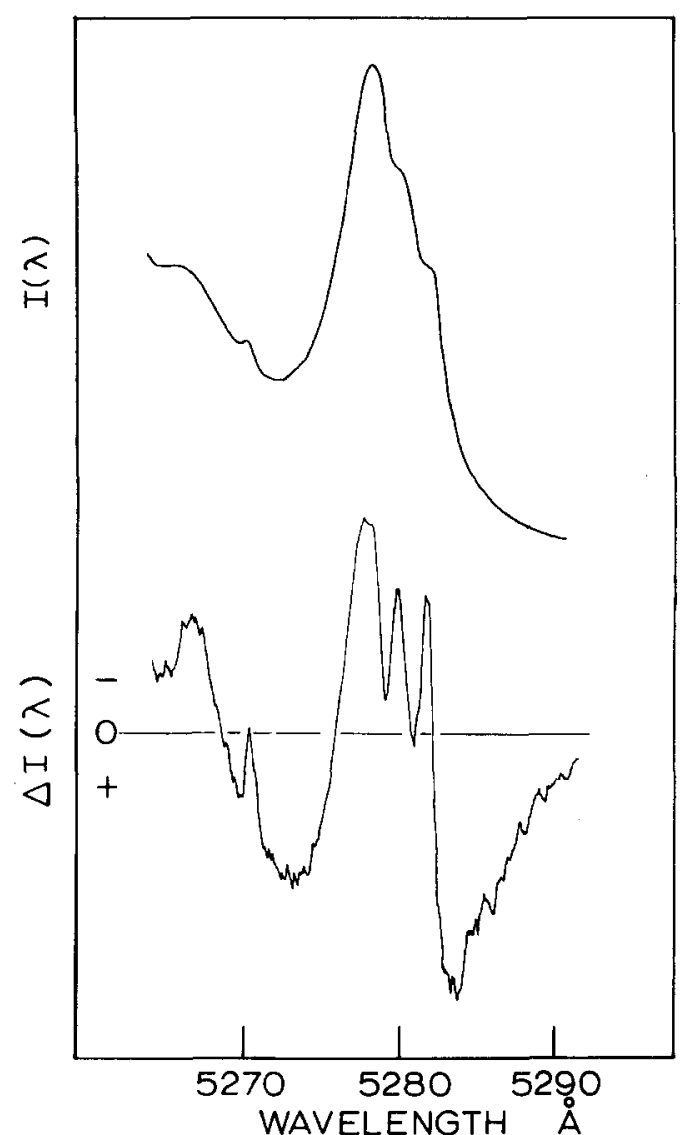

FIG. 2. Upper: Direct absorption spectrum of a feature in the single crystal absorption spectrum of $p$-benzoquinone at $4.2^{\circ} \mathrm{K}$ ambient temperature. Lower: Thermally modulated absorption spectrum obtained at $4.2{ }^{\circ} \mathrm{K}$ ambient temperature and $15 \mathrm{~Hz}$.

purposes. The band group as it appears in the normal absorption spectrum at $4.2^{\circ} \mathrm{K}$ evidently consists of at least three strongly overlapping members of a localized phonon progression with the intensity maximum in the third member. The thermal modulation spectrum shown directly below illustrates the enhancement of the zero-phonon and localized phonon features and the suppression of the phonon background. It should be noted that the thermal modulation spectrum was obtained with identical slit widths and, necessarily, at a slightly higher average temperature than the normal absorption spectrum shown directly above.

The effect of modulation amplitude is illustrated in Fig. 3. Upon further optimizing the ratio $\Delta T / \bar{T}$ by varying the modulation frequency and amplitude, the multiplet is seen to consist of a phonon progression built upon a sharp and intense zero-phonon origin. The members of the progression decline regularly in peak intensity and broaden rapidly in the higher members. The temperature changes in the thermal modulation spectra shown in Figs. 2 and 3 are on the order of $0.1-1^{\circ} \mathrm{K}$.

The spectrum shown in Fig. 4 was obtained from a "molecular impurity" center in a potassium chloride single crystal and consists of a well defined zero-pho- non band (a) and extensive phonon sideband structure. The thermal modulation spectrum shown directly below again illustrates the enhancement of the zero-phonon feature and general suppression of the phonon sideband. We note that certain details of the structure in the phonon sideband differ in their temperature dependence as illustrated in this figure. The feature designated $b$ decreases with increasing lattice temperature, while the feature designated $c$ increases with increasing temperature. The structure of the phonon sideband may correspond to either localized phonon modes or singularities in the phonon density of states. The temperature dependence of the localized phonon modes should follow that of the zero-phonon band and decrease with increasing temperature. We have, therefore quite tentatively correlated features of type $c$ with singularities in the

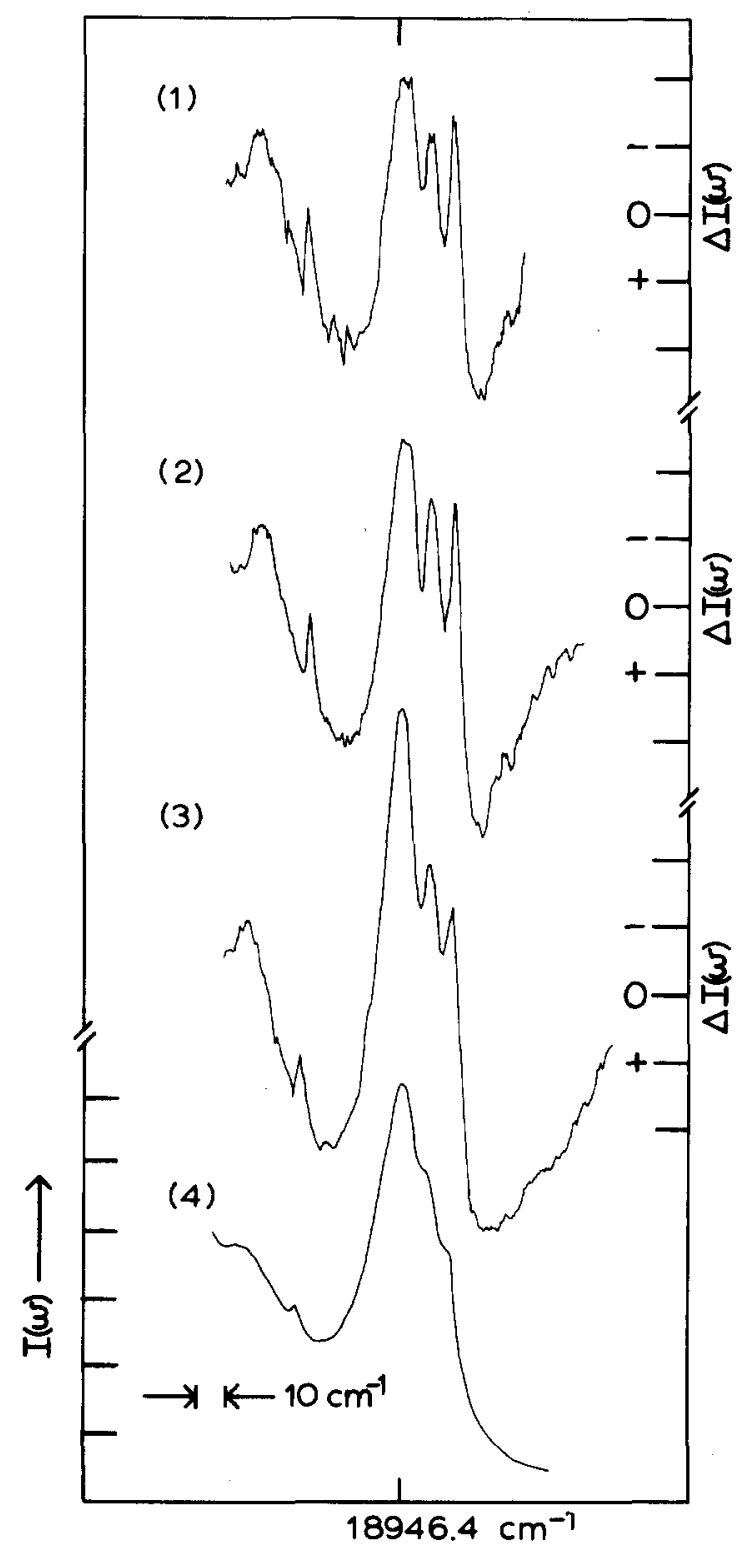

FIG. 3. A series (1-3) of thermal modulation absorption spectra obtained with identical slit width, ambient bath temperature, and modulation frequency, but with increasing heat pulse amplitude. Spectrum (4) is the normal absorption recorded under the same conditions as the modulation spectra. 
phonon density of states and features of type b with localized phonon modes.

The Fig. 4 insert emphasizes the narrowing and small shift of the zero-phonon band in the thermal modulation spectrum. The slight but evident shift with temperature results from modes with both linear and quadratic terms in the adiabatic potential.

In the lower spectrum of Fig. 5 we have illustrated the region of the electronic origin of the dipole forbidden ${ }^{3} B_{1 g} \rightarrow{ }^{1} A_{g}$ transition of $p$-benzoquinone observed in phosphorescence in $p$-dibromobenzene chemically mixed crystal. ${ }^{2}$ The position of the electronic origin is indicated by the vertical line in the figure and will be seen to be the point of approximate "inversion symmetry" in the thermal modulation spectrum shown in the upper trace of the figure. The bands observed to higher and lower frequencies of the electronic origin correspond to anti-Stokes and Stokes transition of one or more localized phonon modes. The anti-Stokes lines increase dramatically with temperature as required by theory, ${ }^{7}$ while the Stokes lines remain essentially unchanged in intensity. Note that there is an overall increase in the

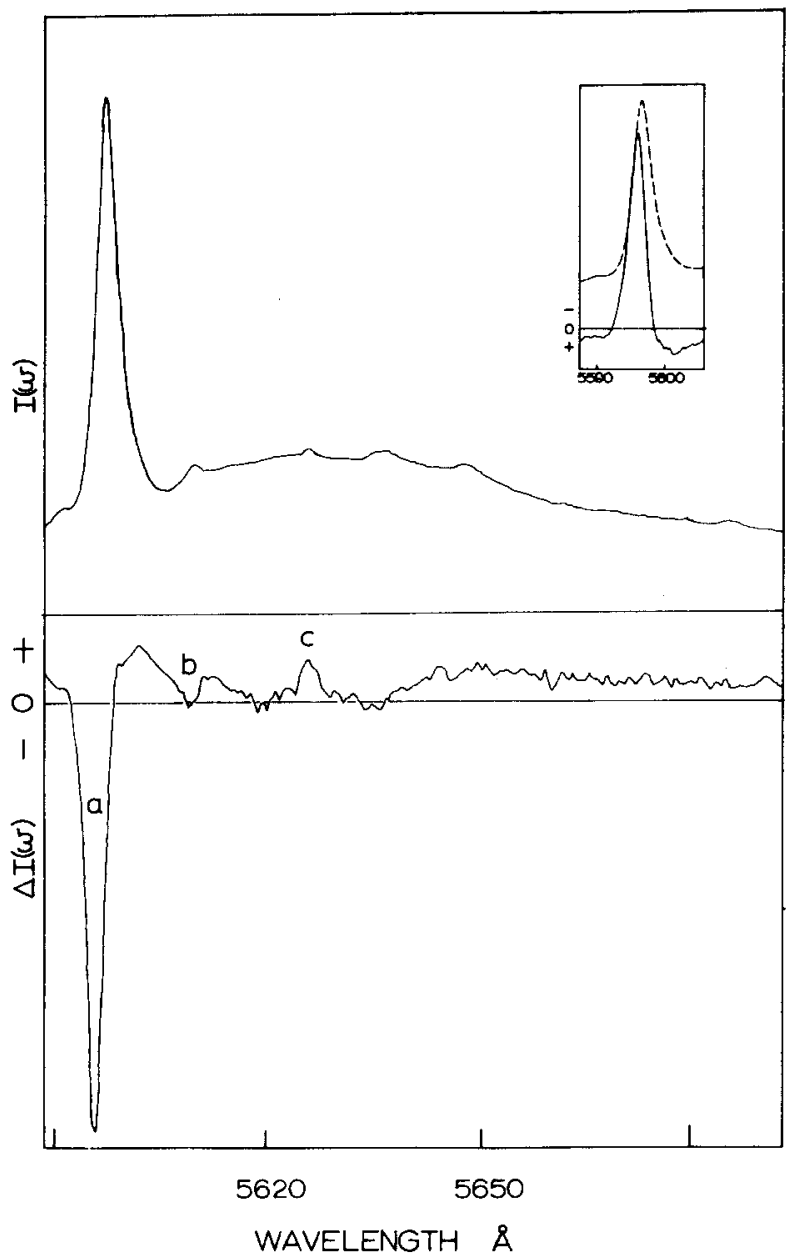

FIG. 4. Experimental line shape (upper spectrum) and thermal modulation line shape (lower spectrum) obtained for a zerophonon vibronic band and phonon sideband in the phosphorescence spectrum of $\mathrm{NO}_{2}^{-}$impurity centers in $\mathrm{KCl}$ single crystals at $5{ }^{\circ} \mathrm{K}$.

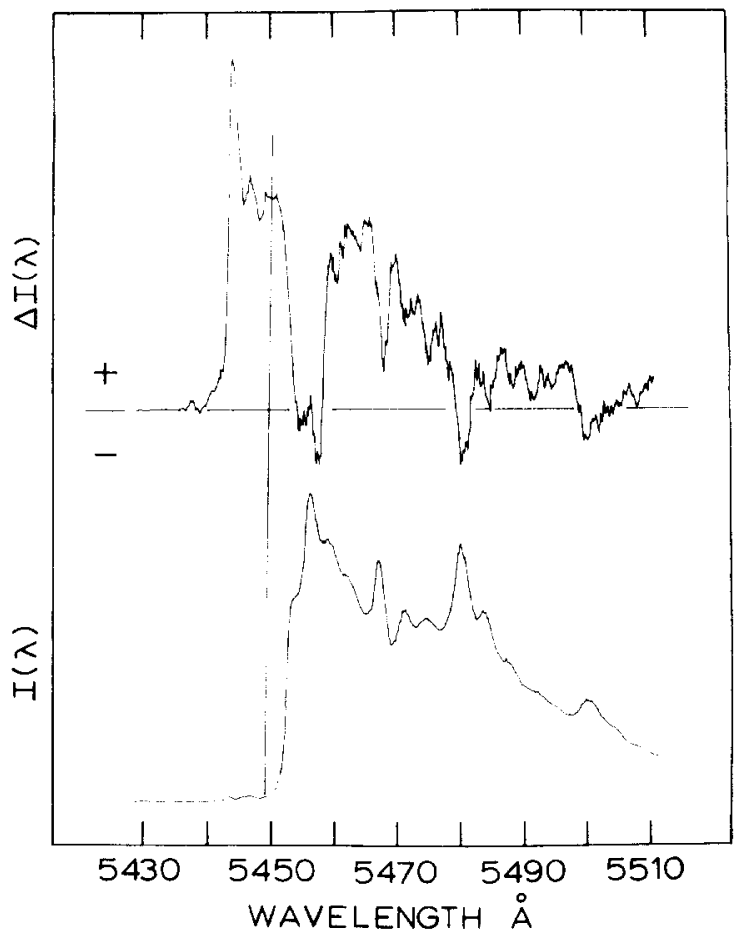

FIG. 5. Upper: Thermally modulated phosphorescence spectrum of $p$-benzoquinone in $p$-dibromobenzene chemically mixed crystal. Lower: Phosphorescence spectrum of $p$-benzoquinone in $p$-dibromobenzene chemically mixed crystal in the region of the electronic origin. The spectrum was recorded at $5{ }^{\circ} \mathrm{K}$ ambient temperature.

integrated intensity of the transition moment on the oscillator coordinates and predicted by Eq. (22). This data is discussed in greater detail in Part II.

\section{ACKNOWLEDGMENTS}

The authors would like to acknowledge partial support for this work from the Air Force Materials Laboratory. One of us (KWH) would like to express his appreciation to the National Science Foundation for support during his tenure as an Energy Related Postdoctoral Fellow.

${ }^{*}$ Part of this work completed in partial fulfillment of the requirements of the $\mathrm{Ph} . \mathrm{D}$. degree at the University of Illinois.

\$Present address: Department of Chemistry, University of Michigan, Ann Arbor, MI 48109.

${ }^{1}$ H. P. Trommsdorff, J. Chem. Phys. 56, 5358 (1972).

${ }^{2}$ A. I. Attia, B. H. Loo, and A. H. Francis, Chem. Phys. Lett. 22, 537 (1973).

${ }^{3}$ S. J. Hunter, H. Parker, and A. H. Francis, J. Chem. Phys. 61,1390 (1974).

${ }^{4}$ A. I. Attia, B. H. Loo, and A. H. Francis, J. Chem. Phys. 61, 4527 (1974).

${ }^{5} \mathrm{M}$. Cardona, Modulation Spectroscopy (Academic, New York, 1969).

${ }^{6}$ See: F. P. Burke and G. J. Small, Chem. Phys. 5, 198 (1974), and references contained therein.

${ }^{7}$ K. K. Rebane, Impurity Spectra of Solids (Plenum, New York, 1970). 
${ }^{8}$ D. L. Dexter, Solid State Phys. 6, 353 (1958).

${ }^{9}$ T. Muto, Prog. Theor. Phys. (Kyoto) 4, 181 (1949).

${ }^{10} \mathrm{~K}$. Huang and A. Rhys. Proc. R. Soc. (London) Ser. A 204, 406 (1950).

${ }^{11} \mathrm{~S}$. Pekar, Zh. Eksperim. Teor. Fiz, 20, 510 (1950).

${ }^{12}$ M. Lax, J. Chem. Phys. 20, 1752 (1952).

${ }^{13}$ R. C. O'Rourke, Phys. Rev. 91, 265 (1953)。
${ }^{14}$ T. H. Keil, Phys. Rev. A 140, 601 (1965).

${ }^{15}$ F. Seitz, Trans. Faraday Soc. 35, 79 (1939).

${ }^{16} \mathrm{~F}$. E. Williams, J. Chem. Phys. 19, 457 (1951).

${ }^{17}$ See, for example, G. Hertzberg, Spectra of Polyatomic

Molecules (Van Nostrand, Princeton, 1964).

${ }^{18}$ G. L. Pollack, Rev. Mod. Phys. 41, 48 (1969).

${ }^{19} \mathrm{H}$. Veenvliet and D. A. Wiersma, Chem. Phys. 8, $432(1975)$. 